\title{
Multicenter analysis of high-resolution computed tomography and positron emission tomography/computed tomography findings to choose therapeutic strategies for clinical stage IA lung adenocarcinoma
}

\author{
Morihito Okada, MD, PhD, ${ }^{\text {a }}$ Haruhiko Nakayama, $\mathrm{MD}, \mathrm{PhD},{ }^{\mathrm{b}}$ Sakae Okumura, MD, PhD, ${ }^{\mathrm{c}}$ \\ Hiromitsu Daisaki, PhD, ${ }^{\mathrm{d}}$ Shuji Adachi, MD, PhD, ${ }^{\mathrm{e}}$ Masahiro Yoshimura, MD, $\mathrm{PhD},{ }^{\mathrm{f}}$ and \\ Yoshihiro Miyata, MD, $\mathrm{PhD}^{\mathrm{a}}$
}

\begin{abstract}
Objective: The detection rates of small lung cancers, especially adenocarcinoma, have recently increased. An understanding of malignant aggressiveness is critical for the selection of suitable therapeutic strategies, such as sublobar resection. The objective of this study was to examine the malignant biological behavior of clinical stage IA adenocarcinoma and to select therapeutic strategies using high-resolution computed tomography, fluorodeoxyglucose-positron emission tomography/computed tomography, and a pathologic analysis in the setting of a multicenter study.
\end{abstract}

\begin{abstract}
Methods: We performed high-resolution computed tomography and fluorodeoxyglucose-positron emission tomography/computed tomography in 502 patients with clinical T1N0M0 adenocarcinoma before they underwent surgery with curative intent. We evaluated the relationships between clinicopathologic characteristics and maximum standardized uptake values on fluorodeoxyglucose-positron emission tomography/computed tomography, ground-glass opacity ratio, and tumor disappearance rate on high-resolution computed tomography and component of bronchioloalveolar carcinoma on surgical specimens, as well as between these and surgical findings. We used a phantom study to correct the serious limitation of any multi-institution study using positron emission tomography/ computed tomography, namely, a discrepancy in maximum standardized uptake values among institutions.
\end{abstract}

Results: Analyses of receiver operating characteristic curves identified an optimal cutoff value to predict highgrade malignancy of 2.5 for revised maximum standardized uptake values, $20 \%$ for ground-glass opacity ratio, $30 \%$ for tumor disappearance rate, and 30\% for bronchioloalveolar carcinoma ratio. Maximum standardized uptake values and bronchioloalveolar carcinoma ratio, tumor disappearance rate, and ground-glass opacity ratio mirrored the pathologic aggressiveness of tumor malignancy, nodal metastasis, recurrence, and prognosis, including disease-free and overall survival.

Conclusions: Maximum standardized uptake value is a significant preoperative predictor for surgical outcomes. High-resolution computed tomography and fluorodeoxyglucose-positron emission tomography/computed tomography findings are important to determine the appropriateness of sublobar resection for treating clinical stage IA adenocarcinoma of the lung. (J Thorac Cardiovasc Surg 2011;141:1384-91)

Earn CME credits at

http://cme.ctsnetjournals.org

\footnotetext{
From the Department of Surgical Oncology, ${ }^{a}$ Hiroshima University, Hiroshima, Japan; Department of Thoracic Surgery, ${ }^{\mathrm{b}}$ Kanagawa Cancer Center, Yokohama, Japan; Department of Thoracic Surgery, ${ }^{\mathrm{c}}$ Cancer Institute Hospital, Tokyo, Japan; Department of Radiology, ${ }^{\mathrm{d}}$ National Cancer Center, Tokyo, Japan; and Department of Radiology ${ }^{\mathrm{e}}$ and Thoracic Surgery, ${ }_{\mathrm{f}}^{\mathrm{f}}$ Hyogo Cancer Center, Akashi, Japan. Disclosures: Authors have nothing to disclose with regard to commercial support.

Received for publication Nov 26, 2010; revisions received Jan 21, 2011; accepted for publication Feb 9, 2011; available ahead of print March 28, 2011.

Address for reprints: Morihito Okada, MD, PhD, Department of Surgical Oncology, Research Institute for Radiation Biology and Medicine, Hiroshima University, 1-2-3-Kasumi, Minami-ku, Hiroshima City, Hiroshima 734-0037, Japan (E-mail: morihito@hiroshima-u.ac.jp).

0022-5223/\$36.00

Copyright (C) 2011 by The American Association for Thoracic Surgery doi: $10.1016 /$ j.jtcvs.2011.02.007
}

The standard treatment for operable non-small cell lung cancer, even early clinical T1N0M0 disease, remains lobectomy with dissection of the hilar and mediastinal lymph nodes. ${ }^{1}$ However, clinical investigations to date have demonstrated that sublobar resection, which chiefly consists of radical segmentectomy with nodal dissection, might be feasible for treating early lung cancers with the tangible advantages of maintained lung function and a reasonable prognosis. ${ }^{2,3}$ To date, there are no means to preoperatively identify early lung cancers that are biologically less aggressive and may be better suited for a sublobar resection.

Recent advances in high-resolution computed tomography (HRCT) and the widespread application of computed tomography (CT) screening have enhanced the discovery of small lung cancers, particularly adenocarcinoma., ${ }^{2,3}$ These early-stage cancers are biologically heterogeneous, and the malignant aggressiveness is not well characterized 


$$
\begin{aligned}
& \text { Abbreviations and Acronyms } \\
& \begin{aligned}
\text { AUC } & =\text { area under the curve } \\
\text { BAC } & =\text { bronchioloalveolar carcinoma } \\
\text { CT } & =\text { computed tomography } \\
\text { DFS } & =\text { disease-free survival } \\
\text { FDG } & =\text { F-18 fluorodeoxyglucose } \\
\text { FOV } & =\text { field of view } \\
\text { GGO } & =\text { ground-glass opacity } \\
\text { HRCT } & =\text { high-resolution computed } \\
& \text { tomography } \\
\text { maxSUV } & =\text { maximum standardized uptake value } \\
\text { PET } & =\text { positron emission tomography } \\
\text { ROC } & =\text { receiver operating characteristic } \\
\text { SUV } & =\text { standardized uptake value } \\
\text { TDR } & =\text { tumor disappearance rate }
\end{aligned}
\end{aligned}
$$

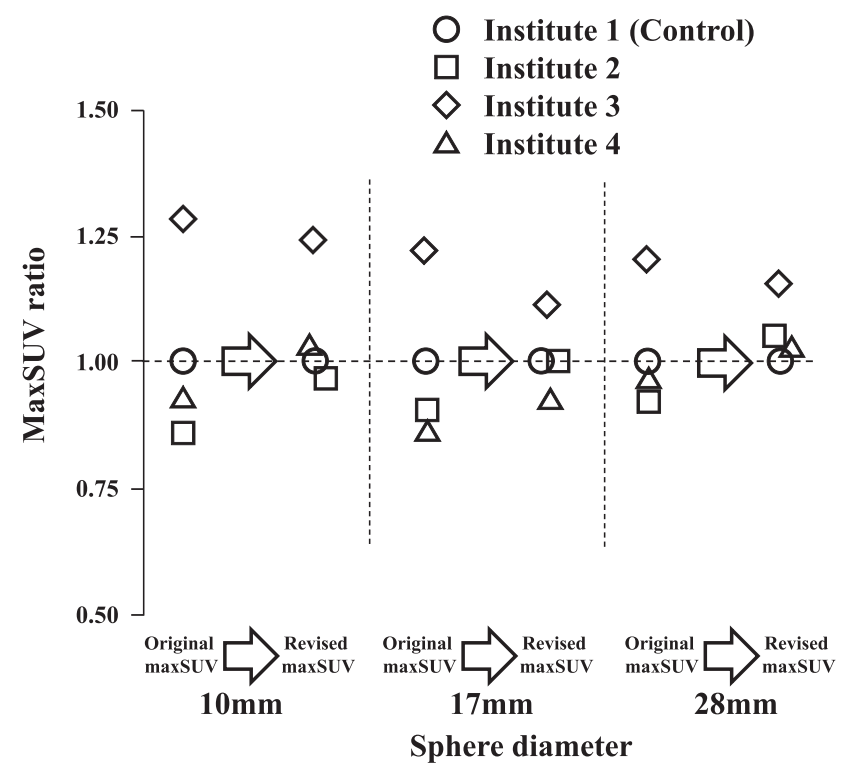

FIGURE 1. maxSUV adjusted using experimental phantom (revised maxSUV). Original maxSUV data were calibrated by multiplying calibration factors based on phantom studies to correct inter-institutional SUV variability. MaxSUV ratio is expressed as maxSUV of each institute for maxSUV of control institute (Institute 1). maxSUV, Maximum standardized uptake value.

HU) and lung (level, -600 HU; width, $1600 \mathrm{HU}$ ) window settings. Ground-glass opacity (GGO) was defined as a misty increase in lung attenuation without obscuring the underlying vascular markings, and we defined the GGO ratio (\%) as [1- (maximum dimension of consolidation of lung windows/maximum dimension of tumor of lung windows) $] \times 100$. Tumor disappearance rate (TDR) $(\%)$ was defined as [1-(tumor area of the mediastinal windows/tumor area of the lung windows) $] \times 100 .^{2,3,10}$

\section{Fluorodeoxyglucose-Positron Emission Tomography/ Computed Tomography}

The patients fasted for more than 4 hours before being intravenously injected with 74-370 MBq of FDG, and then relaxed for at least 1 hour before FDG-PET/CT scanning. Blood glucose was calculated before tracer injection to confirm a level of less than $150 \mathrm{mg} / \mathrm{dL} .^{11}$ Patients with blood glucose values of $150 \mathrm{mg} / \mathrm{dL}$ or greater were excluded from PET/CT acquisition. Images were obtained in this study using Discovery ST (GE Healthcare, Buckinghamshire, England), Aquiduo (Toshiba Medical Systems Corporation, Tochigi, Japan), or Biograph Sensation16 (Siemens Healthcare, South Iselin, NJ) integrated PET/CT scanners. Low-dose unenhanced CT images of a 2- to 4-mm section thickness for attenuation correction and localization of lesions identified by PET were obtained from the head to the pelvic floor of each patient using a standard protocol. Immediately after CT, PET covered the identical axial FOV for 2 to 4 minutes per table position depending on the condition of the patient and scanner perforiterative algorithm with CT-derived attenuation correction. Variations in standardized uptake value (SUV) among institutions were minimized using the anthropomorphic body phantom. A calibration factor was analyzed by dividing the actual SUV by the gauged mean SUV in the phantom background to decrease inter-institutional SUV inconsistencies, and the final maxSUV used in this is referred to as revised maxSUV. The adjustment of the inter-institutional variability in SUV narrowed the range from 0.89 to 1.24 to 0.97 to 1.18 when maxSUV ratio was expressed as the maxSUV of each institute for the maxSUV of the control institute (Figure 1).

\section{High-Resolution Computed Tomography}

Chest images were obtained using 16-row multidetector CT independently of subsequent PET/CT examinations. High-resolution images of the tumors were acquired using the following parameters: $120 \mathrm{kVp}$, $200 \mathrm{~mA}, 1$ - to 2 -mm section thickness, $512 \times 512$ pixel resolution, 0.5 to 1 -second scanning time, a high spatial reconstruction algorithm with a 20-cm field of view (FOV) and mediastinal (level, $40 \mathrm{HU}$; width, 400 mance. All PET images with a 50-cm FOV were reconstructed using an

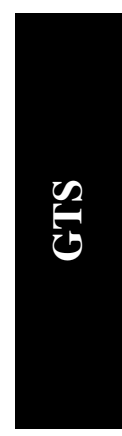

The Journal of Thoracic and Cardiovascular Surgery • Volume 141, Number 6 

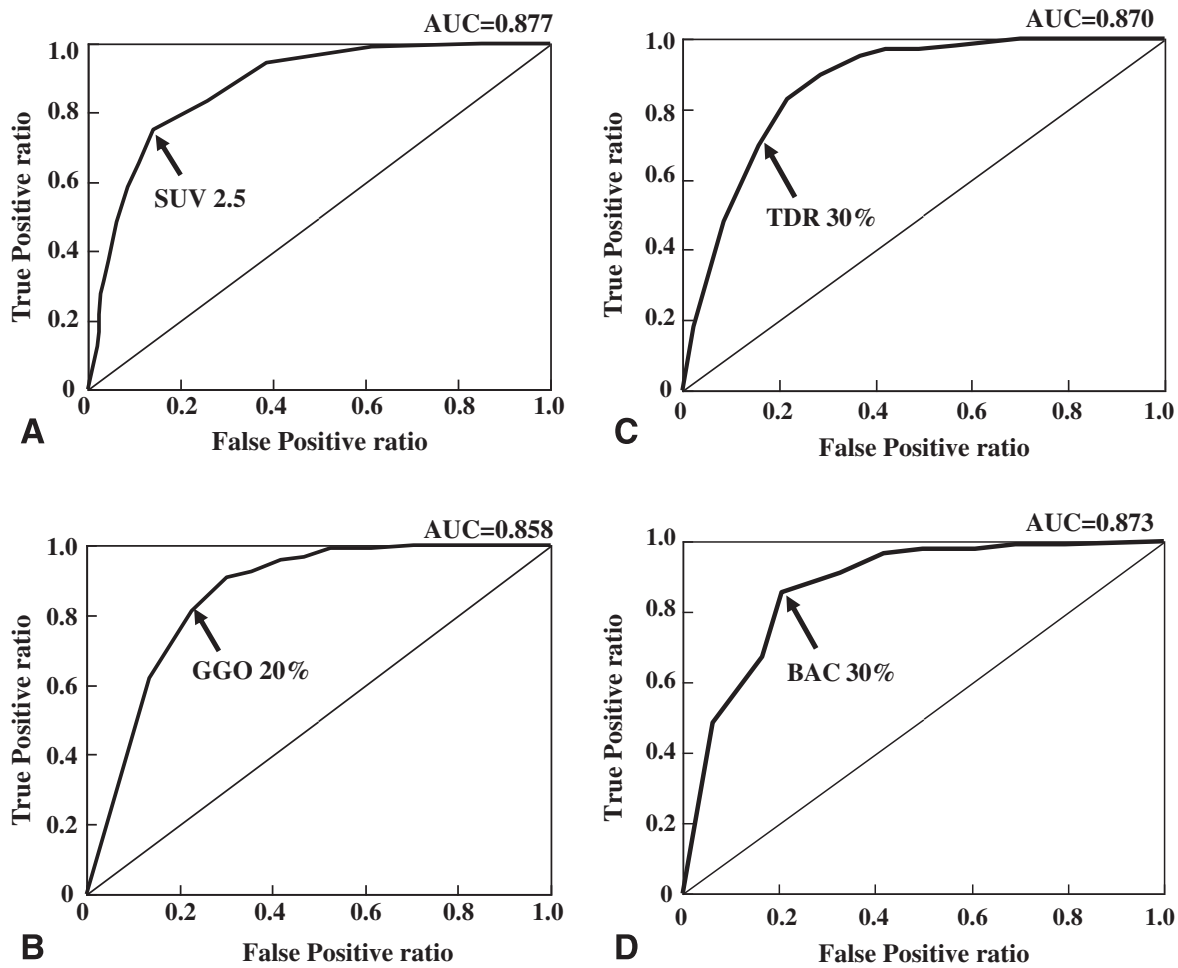

FIGURE 2. ROC curves for positive predictive values of pathologic lymph node metastasis, lymphatic invasion, blood vessel invasion, or pleural invasion. ROC curves are for revised maxSUV (A), GGO ratio (B), TDR (C), and BAC ratio (D). maxSUV, Maximum standardized uptake value; $G G O$, ground-glass opacity; TDR, tumor disappearance rate; $B A C$, bronchioloalveolar carcinoma; $A U C$, area under the curve; SUV, standardized uptake value.

Resected tumors were fixed in $10 \%$ formalin, embedded in paraffin, and pathologically evaluated (including the largest cut) using hematoxylin-eosin and Elastica van Gieson staining. The amount of bronchioloalveolar carcinoma (BAC) areas proportionate to whole tumors was approximately calculated as the BAC ratio. Independent observers and pathologists blindly evaluated GGO and BAC ratios and TDR, and corrected discrepancies among evaluations using mean values.

Receiver operating characteristic (ROC) curves of the revised maxSUV, GGO ratios, TDR, and BAC ratios to predict lymphatic, blood vessel, and pleural invasion or nodal involvement were generated to determine the cutoff values that yielded optimal sensitivity and specificity. The patient population was subdivided on the basis of the cutoff values of the revised SUVmax, GGO, TDR, and BAC ratios derived from ROC curves. We performed multiple logistic regression analyses to demonstrate independent variables of maxSUV, GGO ratio, TDR, and BAC ratio relative to the prediction of pathologic findings for high malignant grade and recurrence. Survival was calculated using the Kaplan-Meier method, and differences were determined using the log-rank test. Disease-free survival (DFS) was defined as time from date of surgery until the first event (relapse or death from any cause) or last follow-up. Overall survival was defined as time from the date of surgery until death from any cause or last follow-up.

\section{RESULTS}

The primary tumor measured by HRCT was $20 \mathrm{~mm}$ or less in diameter in 290 patients $(58 \%)$ and 21 to $30 \mathrm{~mm}$ in 212 patients $(42 \%)$. Lymphatic permeation, blood vessel, and pleural invasion was evident in 76 patients $(15 \%), 92$ patients $(18 \%)$, and 56 patients $(11 \%)$, respectively, and lymph nodes were involved in $38(8 \%)$ of them.
The ROC curves identified optimal revised maxSUV, GGO, TDR, and BAC ratio cutoff values of 2.5 (area under the curve [AUC], 0.877), 20\% (AUC, 0.858), 30\% (AUC, 0.870 ), and $30 \%$ (AUC, 0.873 ), respectively, for predicting pathologic findings of high malignancy (Figure 2). At a cutoff value of 2.5, a high revised maxSUV significantly correlated with male gender, a high CEA value, large tumor, lymphatic invasion, vascular invasion, pleural invasion, and lymph node metastasis (Table 1). Likewise, low TDR, GGO, and BAC ratios were significantly associated with a high CEA value, lymphatic, vascular, pleural invasion, and lymph node metastasis. Possible predictors for lymphatic, vascular, and pleural invasion, and nodal metastasis and recurrence were investigated (Table 2). Revised maxSUV, GGO ratio, TDR, and BAC ratio were all significant determinants for predicting lymphatic permeation, vascular and pleural invasion, nodal metastasis, and recurrence. However, the odds ratios indicated that revised maxSUV and the BAC ratio revealed biological aggressive tumor grade more effectively than GGO ratio and TDR.

A significant difference in DFS was identified (Figure 3) between patients whose adenocarcinoma had revised maxSUV 2.5 or less $(\mathrm{n}=343$; 3-year DFS rate, $96 \%)$ and greater than $2.5(\mathrm{n}=159 ; 3$-year DFS rate, $77 \%$; $P<.001)$. We also found significant differences in DFS between patients whose adenocarcinoma had GGO ratios of 
TABLE 1. Clinical characteristics of patients with cT1N0M0 adenocarcinoma $(n=502)$

\begin{tabular}{|c|c|c|c|c|c|c|c|c|c|}
\hline & \multirow[b]{2}{*}{$\begin{array}{l}\text { Patients } \\
(n=502)\end{array}$} & \multicolumn{2}{|c|}{ Revised maxSUV } & \multicolumn{2}{|l|}{ GGO ratio } & \multicolumn{2}{|l|}{ TDR } & \multicolumn{2}{|l|}{ BAC ratio } \\
\hline & & $\begin{array}{c}\leq 2.5 />2.5 \\
(\mathrm{n}=343 / 159)\end{array}$ & $\begin{array}{c}P \\
\text { value }\end{array}$ & $\begin{array}{l}\geq \mathbf{2 0} \% /<\mathbf{2 0} \% \\
(\mathbf{n}=\mathbf{3 0 4} / \mathbf{1 9 8})\end{array}$ & $\begin{array}{c}P \\
\text { value }\end{array}$ & $\begin{array}{l}\geq \mathbf{3 0} \% /<\mathbf{3 0} \% \\
(\mathbf{n}=\mathbf{3 4 6} / \mathbf{1 5 6})\end{array}$ & $\begin{array}{c}P \\
\text { value }\end{array}$ & $\begin{array}{l}\geq \mathbf{3 0} \% /<\mathbf{3 0} \% \\
(\mathbf{n}=\mathbf{3 0 4} / \mathbf{1 9 8})\end{array}$ & $\begin{array}{c}P \\
\text { value }\end{array}$ \\
\hline $\begin{array}{l}\text { Age }(\mathrm{y}) \\
\quad(\text { means } \pm \mathrm{SD})\end{array}$ & $65.3 \pm 9.6$ & $65.3 \pm 9.4 / 65.5 \pm 9.8$ & .8380 & $65.0 \pm 9.3 / 65.9 \pm 10.0$ & .2790 & $64.7 \pm 9.4 / 66.7 \pm 9.9$ & .0365 & $65.4 \pm 9.5 / 65.3 \pm 9.7$ & .9054 \\
\hline \multicolumn{10}{|l|}{ Gender } \\
\hline Male & 223 & $140 / 83$ & .0169 & $119 / 104$ & .0032 & $144 / 79$ & .0597 & $113 / 110$ & $<.0001$ \\
\hline Female & 279 & $203 / 76$ & & $185 / 94$ & & $202 / 77$ & & $191 / 88$ & \\
\hline \multicolumn{10}{|l|}{ CEA (ng/mL) } \\
\hline$\leq 5$ & 449 & $321 / 128$ & $<.0001$ & $289 / 160$ & $<.0001$ & $324 / 125$ & $<.0001$ & $284 / 165$ & .0003 \\
\hline$>5$ & 53 & $22 / 31$ & & $15 / 38$ & & $22 / 31$ & & $20 / 33$ & \\
\hline \multicolumn{10}{|l|}{ Tumor size (mm) } \\
\hline $\mathrm{T} \leq 20$ & 290 & $218 / 72$ & .0001 & $183 / 107$ & .1723 & $213 / 77$ & .0104 & $180 / 110$ & .4178 \\
\hline$<20 \mathrm{~T} \leq 30$ & 212 & $125 / 87$ & & $121 / 91$ & & $133 / 79$ & & $124 / 88$ & \\
\hline \multicolumn{10}{|l|}{ Ly permeation } \\
\hline positive & 76 & $15 / 61$ & $<.0001$ & $13 / 63$ & $<.0001$ & $25 / 51$ & $<.0001$ & $12 / 64$ & $<.0001$ \\
\hline negative & 426 & $328 / 98$ & & 291/135 & & $321 / 105$ & & $292 / 134$ & \\
\hline \multicolumn{10}{|l|}{$\mathrm{V}$ invasion } \\
\hline positive & 92 & $16 / 76$ & $<.0001$ & $15 / 77$ & $<.0001$ & $23 / 69$ & $<.0001$ & $11 / 81$ & $<.0001$ \\
\hline negative & 410 & $327 / 83$ & & $289 / 121$ & & $323 / 87$ & & 293/117 & \\
\hline \multicolumn{10}{|l|}{ P invasion } \\
\hline positive & 56 & $12 / 44$ & $<.0001$ & $10 / 46$ & $<.0001$ & $14 / 42$ & $<.0001$ & $6 / 50$ & $<.0001$ \\
\hline negative & 446 & $331 / 115$ & & 294/152 & & $332 / 114$ & & 298/148 & \\
\hline \multicolumn{10}{|l|}{$\mathrm{N}$ metastasis } \\
\hline positive & 38 & $10 / 28$ & $<.0001$ & $8 / 30$ & $<.0001$ & $10 / 28$ & $<.0001$ & $5 / 33$ & $<.0001$ \\
\hline negative & 464 & $333 / 131$ & & 296/168 & & $336 / 128$ & & 299/165 & \\
\hline
\end{tabular}

$C E A$, Carcinoembryonic antigen; $L y$, lymphatic; $V$, blood vessel; $P$, pleural; $N$, lymph node; max $S U V$, maximum standardized uptake value; $G G O$, ground-glass opacity; $T D R$, tumor disappearance rate; $B A C$, bronchioloalveolar carcinoma.

TABLE 2. Analysis of possible predictors for Ly factor, $\mathrm{V}$ factor, $\mathrm{P}$ factor, $\mathrm{N}$ factor, and recurrence status

\begin{tabular}{|c|c|c|c|c|}
\hline Factors total $(n=502)$ & Favorable & Unfavorable & Odds ratio $(95 \% \mathrm{CI})$ & $P$ value \\
\hline \multicolumn{5}{|l|}{ Positive Ly factor $(\mathrm{n}=76)$} \\
\hline Revised maxSUV & $\leq 2.5$ & $>2.5$ & $13.61(7.41-25.01)$ & $<.0001$ \\
\hline GGO ratio & $\geq 20 \%$ & $<20 \%$ & $10.45(5.56-19.63)$ & $<.0001$ \\
\hline TDR & $\geq 30 \%$ & $<30 \%$ & $6.24(3.68-10.56)$ & $<.0001$ \\
\hline $\mathrm{BAC}$ ratio & $\geq 30 \%$ & $<30 \%$ & $11.62(6.07-22.25)$ & $<.0001$ \\
\hline \multicolumn{5}{|l|}{ Positive V factor $(\mathrm{n}=92)$} \\
\hline Revised maxSUV & $\leq 2.5$ & $>2.5$ & $18.71(10.37-33.78)$ & $<.0001$ \\
\hline GGO ratio & $\geq 20 \%$ & $<20 \%$ & $12.26(6.78-22.17)$ & $<.0001$ \\
\hline TDR & $\geq 30 \%$ & $<30 \%$ & $11.14(6.57-18.88)$ & $<.0001$ \\
\hline $\mathrm{BAC}$ ratio & $\geq 30 \%$ & $<30 \%$ & $18.44(9.48-35.87)$ & $<.0001$ \\
\hline \multicolumn{5}{|l|}{ Positive $\mathrm{P}$ factor $(\mathrm{n}=56)$} \\
\hline Revised maxSUV & $\leq 2.5$ & $>2.5$ & $10.55(5.39-20.68)$ & $<.0001$ \\
\hline GGO ratio & $\geq 20 \%$ & $<20 \%$ & $8.90(4.37-18.12)$ & $<.0001$ \\
\hline TDR & $\geq 30 \%$ & $<30 \%$ & $8.74(4.60-16.59)$ & $<.0001$ \\
\hline $\mathrm{BAC}$ ratio & $\geq 30 \%$ & $<30 \%$ & $16.78(7.03-40.03)$ & $<.0001$ \\
\hline \multicolumn{5}{|l|}{ Positive $\mathrm{N}$ factor $(\mathrm{n}=38)$} \\
\hline Revised maxSUV & $\leq 2.5$ & $>2.5$ & $7.12(3.36-15.07)$ & $<.0001$ \\
\hline GGO ratio & $\geq 20 \%$ & $<20 \%$ & $6.61(2.96-14.74)$ & $<.0001$ \\
\hline TDR & $\geq 30 \%$ & $<30 \%$ & $7.35(3.47-15.56)$ & $<.0001$ \\
\hline BAC ratio & $\geq 30 \%$ & $<30 \%$ & $11.96(4.58-31.22)$ & $<.0001$ \\
\hline \multicolumn{5}{|c|}{ Positive recurrence $(n=29)$} \\
\hline Revised maxSUV & $\leq 2.5$ & $>2.5$ & $7.71(3.22-18.46)$ & $<.0001$ \\
\hline GGO ratio & $\geq 20 \%$ & $<20 \%$ & $8.25(3.09-22.01)$ & $<.0001$ \\
\hline TDR & $\geq 30 \%$ & $<30 \%$ & $4.66(2.11-10.28)$ & $<.0001$ \\
\hline BAC ratio & $\geq 30 \%$ & $<30 \%$ & $10.84(3.71-31.66)$ & $<.0001$ \\
\hline
\end{tabular}

$L y$, Lymphatic permeation; $V$, blood vessel invasion; $P$, pleural invasion; $N$, lymph node metastasis; max $S U V$, maximum standardized uptake value; $G G O$, ground-glass opacity; $T D R$, tumor disappearance rate; $B A C$, bronchioloalveolar carcinoma. 

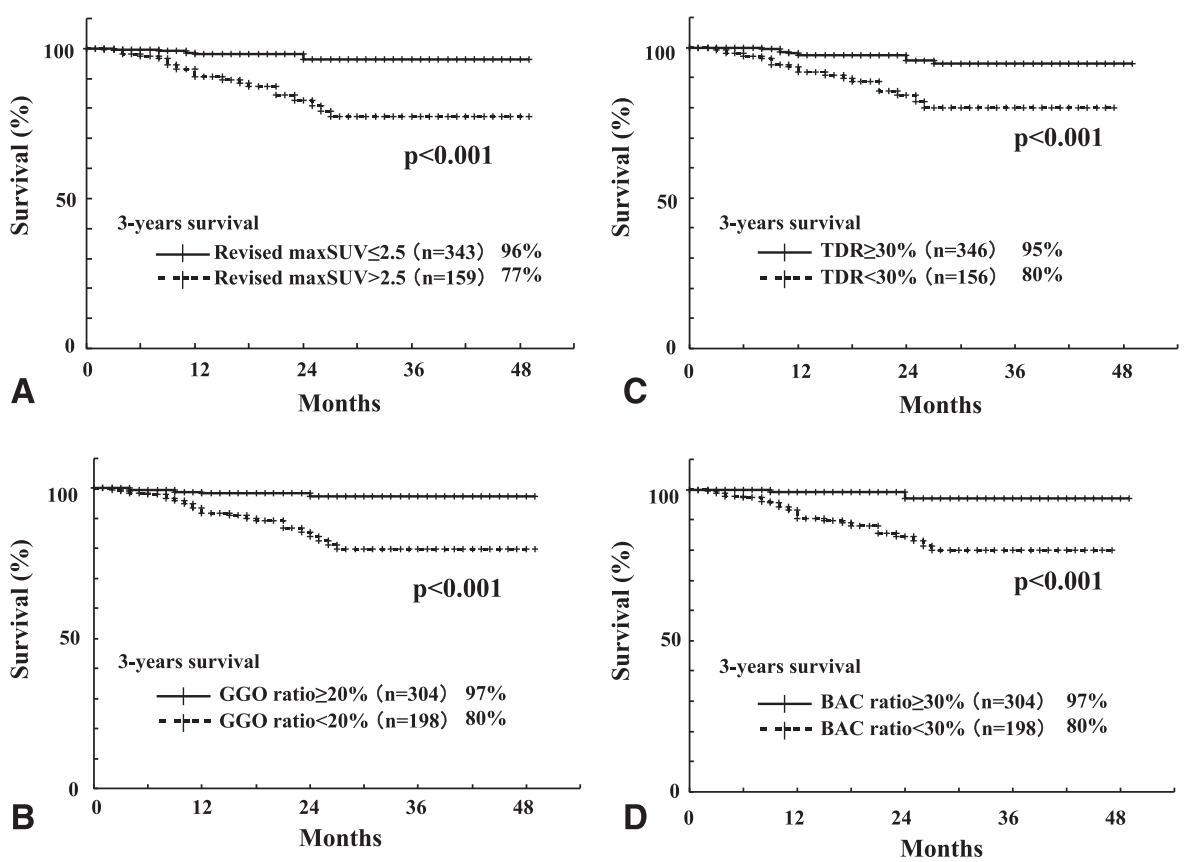

FIGURE 3. DFS curves according to grade of revised maxSUV (A), GGO ratio (B), TDR (C), and BAC ratio (D). maxSUV, Maximum standardized uptake value; $G G O$, ground-glass opacity; $T D R$, tumor disappearance rate; $B A C$, bronchioloalveolar carcinoma.

$20 \%$ or more $(\mathrm{n}=304 ; 3$-year DFS rate, $97 \%)$ and less than $20 \%(\mathrm{n}=198 ; 3$-year DFS rate, $80 \% ; P<.001)$, between those whose adenocarcinoma had TDR $30 \%$ or more $(\mathrm{n}=346 ; 3$-year DFS rate, $95 \%)$ and less than $30 \%$ $(\mathrm{n}=156 ; 3$-year DFS rate, $80 \% ; P<.001)$ and between those whose adenocarcinoma had BAC ratios of $30 \%$ or more $(\mathrm{n}=304$; 3-year DFS rate, 97\%) and less than $30 \%(\mathrm{n}=198 ; 3$-year DFS rate, $80 \% ; P<.001)$. Revised $\operatorname{maxSUV}(P=.016)$ and GGO $(P=.024)$ and pathologic $\mathrm{BAC}(P=.005)$ ratios were significant prognostic factors
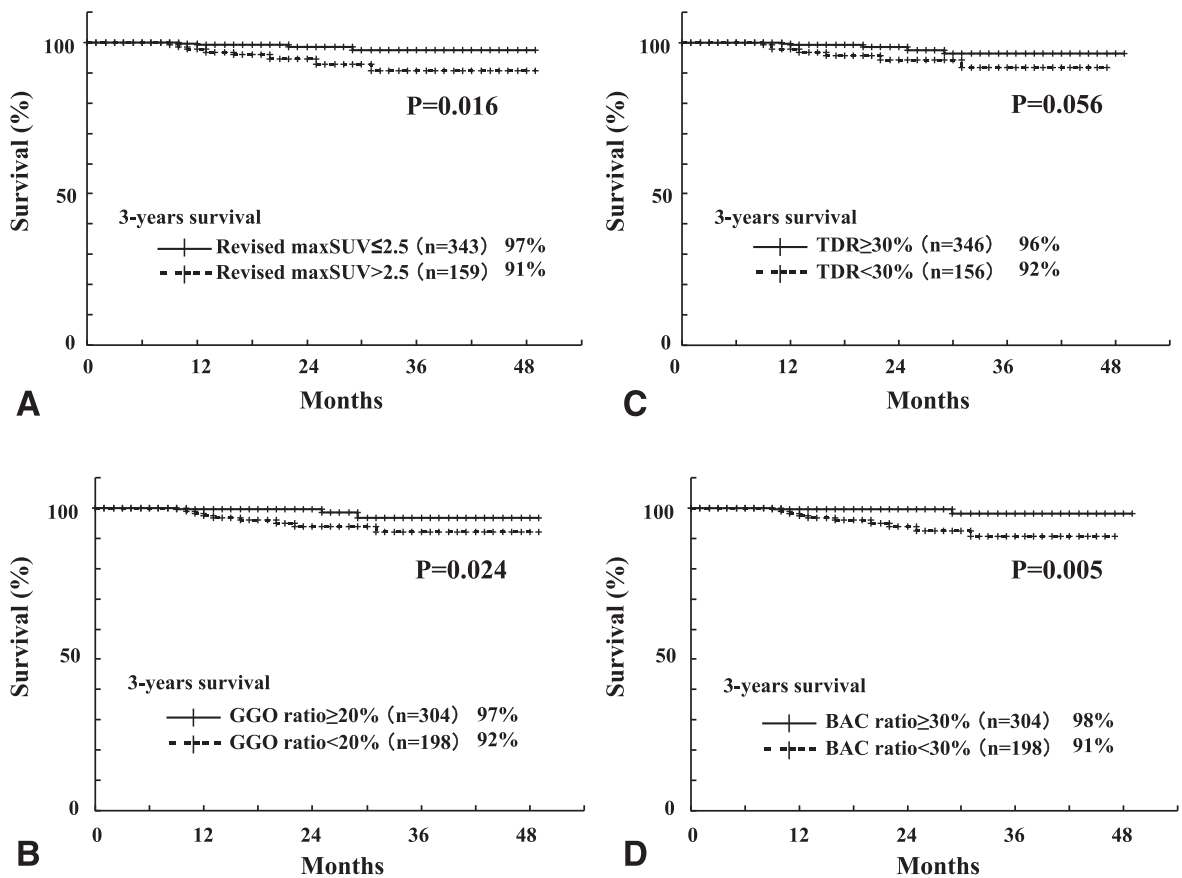

FIGURE 4. Overall survival curves according to grade of revised maxSUV (A), GGO ratio (B), TDR (C), and BAC ratio (D). maxSUV, Maximum standardized uptake value; $G G O$, ground-glass opacity; $T D R$, tumor disappearance rate; $B A C$, bronchioloalveolar carcinoma. 
TABLE 3. Relationship between ground-glass opacity/tumor disappearance rate and revised maxSUV as predictors for $L y$ factor, $\mathrm{V}$ factor, $\mathrm{P}$ factor, $\mathrm{N}$ factor, and recurrence status

\begin{tabular}{|c|c|c|c|c|c|c|}
\hline & $\begin{array}{l}\text { Revised } \\
\text { maxSUV }\end{array}$ & $\begin{array}{c}\text { Ly permeation } \\
(+) /(-)\end{array}$ & $\begin{array}{c}\mathbf{V} \text { invasion } \\
(+) /(-)\end{array}$ & $\begin{array}{c}\text { P invasion } \\
(+) /(-)\end{array}$ & $\begin{array}{c}\text { N metastasis } \\
(+) /(-)\end{array}$ & $\begin{array}{c}\text { Recurrence } \\
(+) /(-)\end{array}$ \\
\hline \multirow{6}{*}{$\begin{array}{l}\text { GGO } \leq 50 \% \text { and } \mathrm{TDR} \leq 50 \% \\
(\mathrm{n}=259)\end{array}$} & $\leq 1.5$ & $2 / 46$ & $1 / 47$ & $3 / 45$ & $0 / 48$ & $0 / 48$ \\
\hline & $(\mathrm{n}=48)$ & $(4 \%)$ & $(2 \%)$ & $(6 \%)$ & $(0)$ & (0) \\
\hline & $<1.5 \leq 2.5$ & $10 / 58$ & $14 / 54$ & $8 / 60$ & $8 / 60$ & $3 / 65$ \\
\hline & $(\mathrm{n}=68)$ & $(15 \%)$ & $(21 \%)$ & $(12 \%)$ & $(12 \%)$ & $(4 \%)$ \\
\hline & $2.5<$ & $59 / 84$ & $73 / 70$ & $43 / 100$ & $27 / 116$ & $22 / 121$ \\
\hline & $(\mathrm{n}=143)$ & $(41 \%)$ & $(51 \%)$ & $(30 \%)$ & $(19 \%)$ & $(15 \%)$ \\
\hline \multirow{6}{*}{$\begin{array}{l}>50 \% \text { GGO or }>50 \% \text { TDR } \\
(\mathrm{n}=243)\end{array}$} & $\leq 1.5$ & $2 / 178$ & $1 / 179$ & $0 / 180$ & $1 / 179$ & $2 / 178$ \\
\hline & $(\mathrm{n}=180)$ & $(1 \%)$ & $(1 \%)$ & (0) & $(1 \%)$ & $(1 \%)$ \\
\hline & $<1.5 \leq 2.5$ & $1 / 46$ & $0 / 47$ & $1 / 46$ & $1 / 46$ & $2 / 45$ \\
\hline & $(n=47)$ & $(2 \%)$ & (0) & $(2 \%)$ & $(2 \%)$ & $(4 \%)$ \\
\hline & $2.5<$ & $2 / 14$ & $3 / 13$ & $1 / 15$ & $1 / 15$ & $0 / 16$ \\
\hline & $(\mathrm{n}=16)$ & $(13 \%)$ & $(19 \%)$ & $(6 \%)$ & $(6 \%)$ & (0) \\
\hline
\end{tabular}

maxSUV, Maximum standardized uptake value; $L y$, lymphatic; $V$, blood vessel; $P$, pleural; $N$, lymph node; $G G O$, ground-glass opacity; $T D R$, tumor disappearance rate.

for overall survival, whereas TDR $(P=.056)$ was marginally significant (Figure 4).

We examined the relationships between HRCT findings and maxSUV for predicting tumor invasiveness, nodal metastasis, and recurrence (Table 3). Generally, solid tumors on HRCT with GGO $50 \%$ or less and TDR $50 \%$ or less had high maxSUV and were more frequently associated with high malignant grade, nodal metastasis, and recurrence. However, solid tumors with lower maxSUV were associated with low malignant grade and far less nodal metastasis and recurrence. Among patients with tumors showing GGO $50 \%$ or less and TDR $50 \%$ or less, $19 \%$ and $15 \%$ of those with a revised maxSUV greater than 2.5 had nodal metastasis and recurrence, respectively,
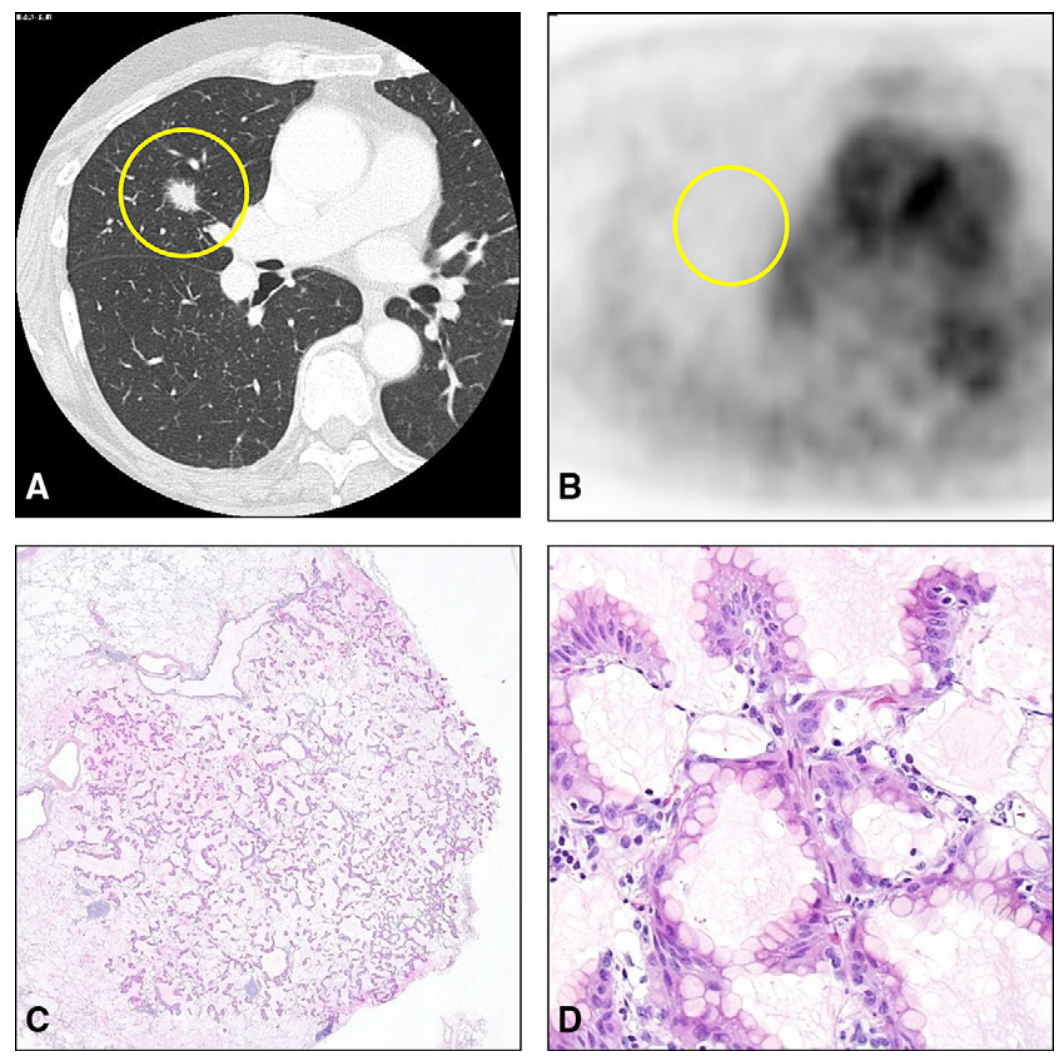

FIGURE 5. Tumor ( $1.5 \mathrm{~cm}$ in diameter) located at right middle lobe. A, HRCT findings show GGO ratio of $5 \%$. B, PET/CT findings show no accumulation. Microscope findings show BAC with mucin formation (C and D, staining with hematoxylin-eosin at $\times 25$ and $\times 200$ magnification, respectively). 

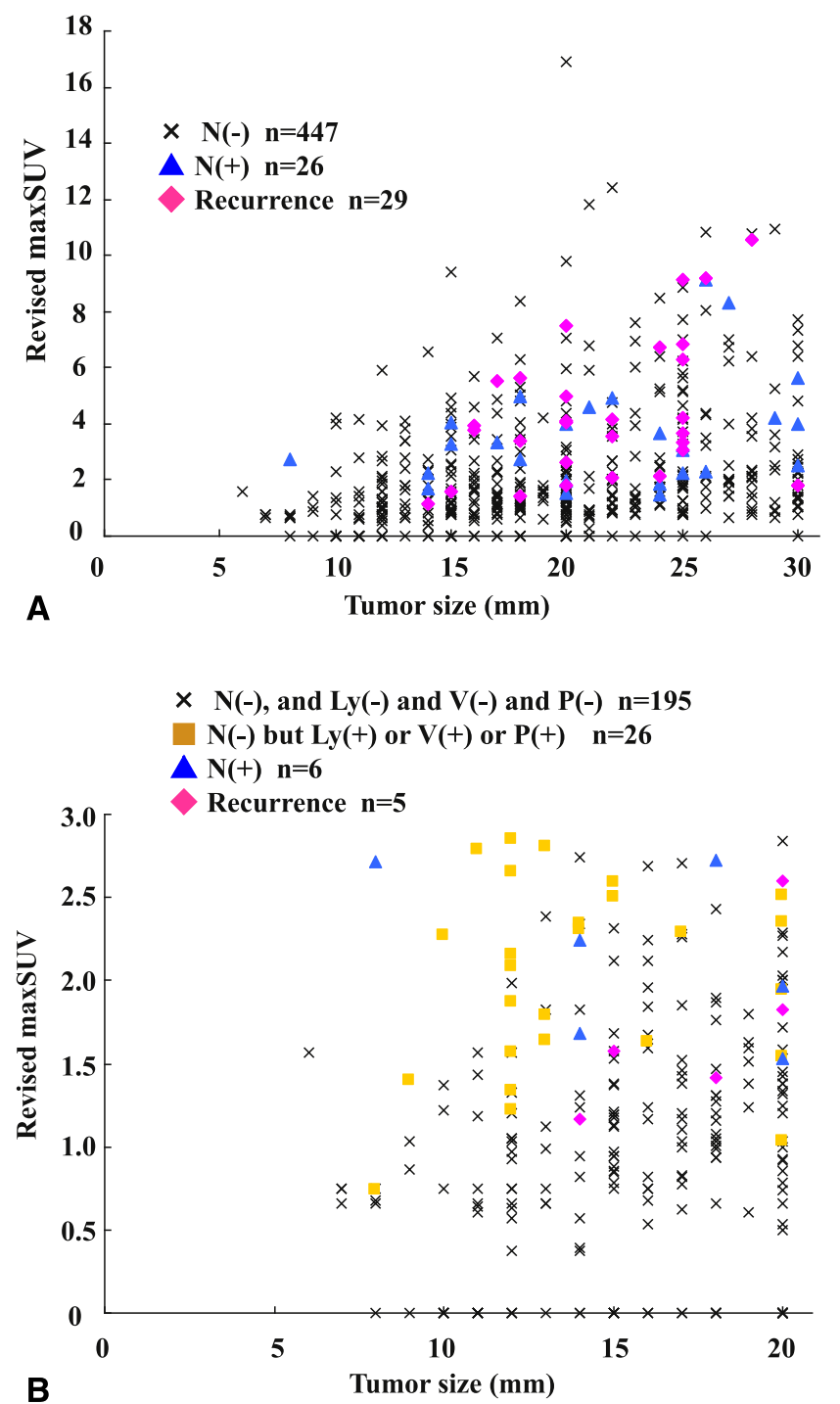

FIGURE 6. Relationship between tumor size and revised maxSUV level in cT1N0M0 adenocarcinomas. A, All patients. B, Tumor size $\leq 20 \mathrm{~mm}$ and revised maxSUV $\leq 3.0$. Nodal metastasis (triangles); recurrence (rhombi); lymphatic, vascular, or pleural invasion without nodal metastasis (squares). max $S U V$, Maximum standardized uptake value; $N$, lymph node; $L y$, lymphatic; $V$, blood vessel; $P$, pleural.

whereas those with tumors showing revised maxSUV 1.5 or less had neither nodal metastasis nor recurrence.

\section{DISCUSSION}

The frequency of identifying small lung cancers has recently increased since $\mathrm{CT}$ and enhanced scanning have become routine procedures and surgeons have thus been able to assess the advantages of sublobar resection, such as radical segmentectomy instead of lobectomy., ${ }^{2,3}$ The biological malignancy of small adenocarcinoma has generally been evaluated on the basis of HRCT findings. The specificity, sensitivity, and accuracy of radiologic diagnoses with HRCT on lymphatic/vessel invasion and nodal involvement of clinical T1NOM0 adenocarcinoma have been examined in a large prospective study (JCOG0201). ${ }^{12}$ The conclusions failed to corroborate predetermined criteria using GGO ratio and TDR for specificity, and thus applicants for radical sublobar resection could not be suitably confirmed on the basis of criteria generated from HRCT findings. Therefore, the development of a novel tool that surpasses the diagnostic ability of HRCT is a current challenge.

Phase III randomized trials of standard lobectomy versus experimental limited resection for small ( $\leq 2 \mathrm{~cm}$ in diameter) peripheral non-small cell lung cancers are ongoing in the United States (CALGB-140503) and in Japan (JCOG0802/ WJOG4607L). Because the biological behavior of several types of small lung cancers is aggressive, to detect indolent lung cancers preoperatively with radiographic imaging is important for the standardization of sublobar resection, although accurate radiographic definition of tumor extension and metastasis is challenging. We previously demonstrated that maxSUV on FDG-PET/CT is a potentially promising parameter of the degree of malignancy among cT1N0M0 lung adenocarcinoma. ${ }^{7,8}$ However, relatively few patients were analyzed and postoperative survival data were obtained during a short period. We therefore performed an extended, larger multicenter study. To our knowledge, a large scale multi-institutional study of malignant tumor grade analyzed using PET/CT has not been reported.

The present analyses revealed that adenocarcinoma with high maxSUV and low GGO ratio, TDR, or BAC ratio is more closely associated with lymphatic, vessel, pleural invasion, lymph node metastasis, and recurrence. We also found a closer association between serum CEA levels and maxSUV, GGO ratio, TDR, and BAC ratio, which is preoperatively vital in uncovering patients at high risk of potential advanced disease. ${ }^{13,14}$ High maxSUV was also a poor prognostic determinant for DFS and overall survival. These outcomes suggested that maxSUV can be a realistic surrogate marker of tumor malignancy grade and that FDG-PET/CT in addition to HRCT is a potent prognostic modality with which to detect patients at high risk of recurrence after curative resection of small adenocarcinoma.

Because PET/CT imaging reflects metabolic activity as glucose metabolism whereas HRCT generates only anatomic images, findings of PET/CT could be more powerful clinical predictors of biological tumor performance than those of HRCT. Most solid tumors on HRCT have high maxSUV and aggressive malignant behavior, whereas solid tumors with lower maxSUV were less aggressive. Figure 5 shows a mucinous BAC with indolent behavior that appeared as a solid tumor on HRCT with no accumulation on PET/CT.

We analyzed the association between tumor size and maxSUV in cT1NOM0 adenocarcinoma (Figure 6). A larger 
tumor was associated with a higher maximum SUV, and both size and maxSUV can predict tumor invasiveness, nodal metastasis, and recurrence. The figures show a notable tendency of small adenocarcinoma with high maxSUV to have more malignant features than larger adenocarcinoma with low maxSUV.

One of the major limitations of multicenter trials using PET is the wide variation in SUV among institutions. Many factors, such as preparation procedures, scan acquisition, image reconstruction, and data analysis affect SUV ${ }^{15,16}$ although semiquantitative SUV in patients with cancer is extensively applied to diagnose, stage, and evaluate therapeutic effectiveness. ${ }^{4-6}$ Westerterp and colleagues ${ }^{17}$ reported that a discrepancy in SUV of up to $30 \%$ among institutions could create a significant problem for multicenter studies. Inter-institutional variability in SUV obtained in the present study was minimized using an anthropomorphic body phantom. Therefore, quantitative SUVs adjusted by phantom studies can be dependable, and such correction will help to overcome one major limitation of multicenter of PET studies.

\section{CONCLUSIONS}

The findings of HRCT and FDG-PET/CT are important to select therapeutic strategies for treating c-stage IA adenocarcinoma of the lung, and maxSUV on FDG-PET/CT is an especially significant preoperative predictor for surgical outcomes. Sublobar resection might be performed in patients with adenocarcinoma with maxSUV 1.5 or less, and lymphadenectomy is required for tumors with maxSUV greater than 1.5 .

\section{References}

1. Ginsberg RJ, Rubinstein LV. Randomized trial of lobectomy versus limited resection for T1N0 non-small cell lung cancer. Lung Cancer Study Group. Ann Thorac Surg. 1995;60:615-22.

2. Okada M, Koike T, Higashiyama M, Yamato Y, Kodama K, Tsubota N. Radical sublobar resection for small-sized non-small cell lung cancer: a multicenter study. J Thorac Cardiovasc Surg. 2006;132:769-75.

3. Nakayama H, Yamada K, Saito H, Oshita F, Ito H, Kameda Y, et al. Sublobar resection for patients with peripheral small adenocarcinomas of the lung: surgical outcome is associated with features on computed tomographic imaging. Ann Thorac Surg. 2007;84:1675-9.

4. Pieterman RM, van Putten JW, Meuzelaar JJ, Mooyaart EL, Vaalburg W, Koëter GH, et al. Preoperative staging of non-small-cell lung cancer with positron-emission tomography. N Engl J Med. 2000;343:254-61.

5. Vansteenkiste J, Fischer BM, Dooms C, Mortensen J. Positron-emission tomography in prognostic and therapeutic assessment of lung cancer: systematic review. Lancet Oncol. 2004;5:531-40.

6. Juweid ME, Cheson BD. Positron-emission tomography and assessment of cancer therapy. N Engl J Med. 2006;354:496-507.

7. Okada M, Tauchi S, Iwanaga K, Mimura T, Kitamura Y, Watanabe H, et al. Associations among bronchioloalveolar carcinoma components, positron emission tomographic and computed tomographic findings, and malignant behavior in small lung adenocarcinomas. J Thorac Cardiovasc Surg. 2007;133 1448-54.

8. Nakayama H, Okumura S, Daisaki H, Kato Y, Uehara H, Adachi S, et al. Value of integrated positron emission tomography revised using a phantom study to evaluate malignancy grade of lung adenocarcinoma: a multicenter study. Cancer. 2010;116:3170-7.

9. Mawlawi O, Podoloff DA, Kohlmyer S, Williams JJ, Stearns CW, Culp RF, et al. Performance characteristics of a newly developed PET/CT scanner using NEMA standards in 2D and 3D modes. J Nucl Med. 2004;45: 1734-42.

10. Shimizu K, Yamada K, Saito H, Noda K, Nakayama H, Kameda Y, et al. Surgically curable peripheral lung carcinoma. Correlation of thin-section CT findings with histologic prognostic factors and survival. Chest. 2005;127: 871-8.

11. Delbeke D, Coleman RE, Guiberteau MJ, Brown ML, Royal HD, Siegel BA, et al. Procedure guideline for tumor imaging with 18F-FDG PET/CT 1.0 J Nucl Med. 2006;47:885-95.

12. Suzuki K, Koike T, Shibata T, Kusumoto M, Asamura H, Nagai K, et al. Evaluation of radiologic diagnosis in peripheral clinical IA lung cancers. A prospective study for radiological diagnosis of peripheral early lung cancer (JCOG0201). J Clin Oncol. 2006;24:7220.

13. Okada M, Nishio W, Sakamoto T, Uchino K, Yuki T, Nakagawa A, et al. Prognostic significance of perioperative serum carcinoembryonic antigen in nonsmall cell lung cancer: analysis of 1,000 consecutive resections for clinical stage I disease. Ann Thorac Surg. 2004;78:216-21.

14. Okada M, Nishio W, Sakamoto T, Uchino K, Yuki T, Nakagawa A, et al. Effect of histologic type and smoking status on interpretation of serum carcinoembryonic antigen value in non-small cell lung carcinoma. Ann Thorac Surg. 2004;78: 1004-9.

15. Boellaard R, Oyen WJG, Hoekstra CJ, Hoekstra OS, Visser EP, Willemsen AT, et al. The Netherlands protocol for standardisation and quantification of FDG whole body PET studies in multi-centre trials. Eur J Nucl Med Mol Imaging. 2008;35:2320-33.

16. Boellaard R, Krak NC, Hoekstra OS, Lammertsma AA. Effects of noise, image resolution, and ROI definition on the accuracy of standard uptake values: a simulation study. J Nucl Med. 2004;45:1519-27.

17. Westerterp M, Pruim J, Oyen W, Hoekstra O, Paans A, Visser E, et al. Quantification of FDG PET studies using standardized uptake values in multicenter trials: effects of image reconstruction, resolution and ROI definition parameters. Eur J Nucl Med Mol Imaging. 2007;34:392-404. 